\title{
Um Ambiente Virtual onde a Educação a Distância Encontra- se com a Educação Matemática Inclusiva
}

\author{
Carlos Eduardo Rocha dos Santos ${ }^{* 1}$, Solange Hassan Ahmad Ali Fernandes ${ }^{2}$ \\ 1 Doutorando, Universidade Anhanguera. Rua Maria Cândida, 1813 - São Paulo - SP - Brasil. \\ carlao_santos@yahoo.com.br \\ 2 Professor, Universidade Anhanguera. Rua Maria Cândida, 1813 - São Paulo - SP - Brasil. \\ solangehf@gmail.com
}

\section{Resumo}

O presente artigo visa apresentar a trajetória de uma pesquisa de doutoramento, ainda em desenvolvimento, que tem como objetivo principal apresentar um ambiente virtual de aprendizagem (AVA) que seja acessível e que possa atender principalmente pessoas com necessidades especiais no campo sensorial da visão e da audição. Pesquisas apontam que os AVA disponíveis em sua grande maioria não são acessíveis, problema que se estende ao material didático disponível nos cursos a distância. Diante dessa preocupação, estamos trabalhando com a perspectiva de que, ao final de nossa trajetória, consigamos apresentar um ambiente acessível, de forma a tornar igualitárias as chances de acesso ao ensino, principalmente, superior. Para alcançar nossos objetivos estamos nos valendo do uso da pesquisa-ação como metodologia de ensino, pois queríamos, a partir das vozes dos participantes, colher informações para realizar o design de nosso AVA. Essas informações foram obtidas através de um questionário on-line que foi respondido por pessoas com limitações sensoriais visuais e auditivas. Essas pessoas tiveram a oportunidade de explorar um AVA utilizado em pesquisa anterior, que apresentava preocupação semelhante e, ao final, foram convidadas a responder a um questionário, onde puderam nos dar sugestões de melhorias. A partir dessas informações, realizamos o redesign do AVA, que ainda será testado com a oferta de um curso piloto. Queremos, ao final, apresentar uma alternativa ao ensino presencial que possa atender a todos, mostrando quais as características que o AVA, a tutoria e o material didático devem possuir para garantir um ensino de qualidade.

Palavras-chave: Inclusão; Necessidades especiais; Educação a distância; Ambiente virtual de aprendizagem; Tecnologia. 


\title{
A virtual Environment where the Distance Education Meets Inclusive Education in Mathematics
}

\begin{abstract}
This article presents the trajectory of a doctoral research project, still under development, which aims to present a virtual learning environment (VLE) that is accessible and can mainly serve persons with special needs in the sensory field of vision and hearing. Surveys show that VLE available mostly are not accessible--a problem that extends to the teaching material available in distance learning courses. Given this concern, we are working with the prospect that at the end of our journey we will succeed in presenting an accessible environment, in order to make equal chances of access to education--especially higher. To achieve our goals it is costing us the use of action research as a teaching methodology because we wanted to collect information from the participants to perform the design of our VLE. This information was collected through an online questionnaire that was answered by people with visual and auditory sensory limitations. These people had the opportunity to explore a VLE used in previous research, which showed similar concern, and at the end were asked to answer a questionnaire, which could give us suggestions for improvements. From this information we conducted the redesign of the VLE, which will still be tested with the offer of a pilot course. In the end we want to present an alternative to classroom learning that can suit everyone, showing what features the VLE, mentoring, and courseware must possess to ensure a quality education for all.
\end{abstract}

Keywords: Inclusion; Special needs; Distance education; Virtual learning environment; Technology. 


\section{Introdução}

Vivemos em uma sociedade que produz inovações tecnológicas e informações freneticamente, mas tais ferramentas e informações não chegam a todas as pessoas da mesma maneira. É o que ocorre no caso das pessoas com necessidades educacionais especiais e, particularmente, com aqueles que têm limitações visuais ou auditivas.

Por outro lado, uma das características da tecnologia é promover a inclusão das pessoas que possuem algum fator de limitação facilitando sua integração à sociedade. Criar possibilidades para que as pessoas cegas ou surdas possam estudar, interagir, aperfeiçoar-se, capacitar-se e, acima de tudo, sentir-se inseridas no contexto educacional foi o que nos motivou a realizar nossa pesquisa.

No Brasil, as pesquisas indicam aumento significativo no número de estudantes que migraram da educação especial para as classes comuns do ensino regular. 0 percentual de alunos da educação especial em escolas regulares cresceu $41 \%$ em 10 anos. São 375.775 estudantes incluídos em classes comuns. De acordo com o Censo Escolar da Educação Básica (INEP, 2013), em 2007, os alunos deficientes em classes especiais e escolas exclusivas somavam 306.136 passando para 194.421 em 2013, caracterizando uma queda de $36,49 \%$. Em contrapartida, os alunos deficientes inseridos em classes comuns passaram de 348.470, em 2007, para 648.921, em 2013, ou seja, um aumento de $86,22 \%$. Esses números apontam que a presença de alunos com necessidades educacionais especiais compondo o cenário escolar é uma realidade.

Diante desse contexto, acreditamos que fazendo o uso da tecnologia, em especial, a educação a distância on-line através de ambientes virtuais de aprendizagem (AVA), poderemos propiciar alternativas de estudos à educação presencial e, provavelmente, promover meios de integração das pessoas com limitações sensoriais e físicas, principalmente, no mercado de trabalho.

Quando o assunto é ambiente virtual, um fator de suma importância é o da acessibilidade. Porém, nossas investigações apontam que a maioria dos sites disponíveis na rede mundial de computadores não possuem adaptações para atender às necessidades dos usuários com limitações sensoriais. Tornar a web acessível significa disponibilizar diversos conteúdos de forma flexível a grupos de usuários 
heterogêneos. Um site só pode ser considerado eficaz a partir do instante em que qualquer pessoa possa ter acesso ao seu conteúdo com a mesma eficácia.

Nesse sentido, nosso principal objetivo é planejar, desenhar e manter um ambiente virtual que atenda as pessoas com necessidades especiais com o intuito de oferecer cursos de Educação a Distância acessível à diversidade de usuários que compõem o quadro discente. Em nossa pesquisa, chamaremos de EaDonline, assim como Borba, Malheiros e Zullatto (2007), que definem:

Eadonline pode ser entendida como a modalidade de educação que acontece primordialmente mediada por interações via internet $e$ tecnologias associadas. Cursos e disciplinas cuja interação aconteça utilizando interfaces como salas de bate-papo, videoconferências, fóruns, etc. se encaixam nessa modalidade. (IBID, p. 15).

Construir uma sociedade para todos implica na conscientização coletiva da diversidade humana (nacionalidade, sexo, religião, sexualidade, cor, idade, raça e deficiência ou outras) e na estruturação para atender às necessidades de cada cidadão. Certamente a escola tem um papel fundamental nessa construção e a inserção de educandos com necessidades educacionais especiais, no meio escolar, representa uma forma de tornar a sociedade mais democrática (Martins, 2002).

\section{Metodologia}

Um dos desígnios de nossa pesquisa ${ }^{1}$ é envolver aqueles que necessitam de ferramentas especiais para ter acesso à Educação a Distância adotando uma metodologia de pesquisa ação. Mais especificamente, pretendeu-se conduzir uma investigação cogenerativa com as características apontadas por Greenwood e Levin (2000, p. 96):

1. Pesquisa ação é uma investigação na qual, participantes e pesquisadores cogeram conhecimento através de um processo de comunicação colaborativa na qual todas as contribuições dos participantes são levadas a sério. Os significados construídos no processo de investigação conduzem à ação social, ou essas reflexões sobre a ação conduzem à construção de novos significados;

2. a pesquisa ação trata a diversidade de experiências e capacidades dentro do grupo local como uma oportunidade para o enriquecimento para o processo de pesquisa/ação;

3. pesquisa ação produz resultados válidos de pesquisa; 
4. pesquisa ação centra-se no contexto e objetiva resolver os problemas da vida real no contexto.

Em outras palavras, optamos por um tipo de pesquisa qualitativa em que usuários e pesquisadores têm a oportunidade de cogerar conhecimento por um processo de comunicação colaborativa. Desse modo, a investigação cogenerativa que pretendemos conduzir poderá gerar novos significados que por sua vez conduzam a uma ação social ou à reflexão sobre a ação.

Mais especificamente, a pesquisa-ação que nos propomos conduzir de acordo com o proposto por Greenwood e Levin (2000, pp. 94-95) é um tipo de pesquisa social com base empírica que é concebida e realizada em estreita associação com uma ação ou com a solução de um problema coletivo no qual os pesquisadores e os participantes representativos da situação ou problema estão envolvidos de modo participativo e cooperativo.

Iniciaremos esta pesquisa estabelecendo metas e objetivos comuns entre nossos interesses e os interesses dos usuários de cursos de Educação a Distância, ou seja, pesquisadores e usuários passarão a compor um grupo com objetivos e metas comuns, interessados nos problemas que emergem no contexto da Educação a Distância.

Uma pesquisa do tipo pesquisa ação, na qual há a necessidade de se conhecer com profundidade os problemas do contexto, exige que se considere uma variedade de instrumentos para coletas de dados. A diversidade das intervenções que realizaremos durante a pesquisa e o tipo de análises pretendidas são fatores determinantes para a escolha das técnicas e dos instrumentos usados nas coletas de dados.

Estruturamos nossa pesquisa em quatro ciclos, cada um com duas fases.

\section{○ Ciclo I - Caracterização da situação atual}

Fase I - Levantamento das barreiras encontradas por usuários com necessidades educacionais especiais para o acompanhamento de cursos realizados a distância.

Nessa fase, utilizamos de três recursos para realizar o levantamento de dados. O primeiro se baseou nos resultados de nossa pesquisa de mestrado, que trouxe importantes sugestões de melhorias para nosso ambiente virtual. O segundo recurso 
utilizado foi um questionário que disponibilizamos às pessoas cegas e surdas que, após navegarem e explorarem o ambiente virtual utilizado na pesquisa anterior, puderam responder algumas perguntas, apontando sugestões de melhorias, críticas e novas ideias para deixar o ambiente totalmente acessível. Por último, mas não menos importante, utilizamos informações de outros trabalhos que trataram da acessibilidade de sites e ambientes virtuais, para isso, fizemos uma extensa revisão bibliográfica.

Fase II - Organização e análise dos dados coletados nas entrevistas

Nessa fase, organizamos e analisamos os dados coletados. Esses dados nos ofereceram parâmetros para que pudéssemos viabilizar e avaliar a acessibilidade do AVA sobre o qual estamos trabalhando, nos fornecendo, assim, informações para a organização do Ciclo II.

\section{Ciclo II - Design do AVA}

\section{Fase I - Design Universal}

Nessa fase, iniciamos o design do AVA centrando-nos inicialmente nas questões referentes à acessibilidade, usabilidade e adaptabilidade.

São sete os princípios do desenho universal (Cud, 2012 apud Fernandes et al, 2013, pp. 24-25):

1. Equiparação nas possibilidades de uso: O desenho é útil às pessoas com habilidades diferenciadas oferecendo os mesmos meios de uso para todos os usuários.

2. Flexibilidade no uso: Atende a uma ampla gama de indivíduos, preferências e habilidades proporcionando escolha dos métodos de utilização.

3. Uso simples e intuitivo: $O$ uso é de fácil compreensão, independentemente de experiência, de nível de formação e da capacidade de concentração do usuário. 
4. Captação da informação: O desenho comunica eficazmente ao usuário as informações necessárias, independentemente de sua capacidade sensorial ou de condições ambientais usando diferentes modos (pictórico, verbal, tátil) para apresentação de informações essenciais e é compatível com uma variedade de técnicas ou dispositivos utilizados por pessoas com limitações sensoriais.

5. Tolerância ao erro: Minimiza o risco e as consequências adversas de ações involuntárias ou imprevistas.

6. Mínimo esforço físico: Pode ser utilizado com pouco esforço e de forma eficiente, minimizando ações repetitivas e favorecendo uma postura corporal confortável.

7. Dimensão e espaço para uso e interação: Oferece espaços e dimensões apropriados para interação, alcance, manipulação e uso, independentemente de tamanho, postura ou mobilidade do usuário.

Em cada um desses sete princípios, pode-se perceber a preocupação em garantir que toda e qualquer pessoa possa ter acesso a produtos e serviços. No âmbito da web, acreditamos que o desenho universal proporciona equiparação de oportunidade de acesso, podendo ser apontado como uma possibilidade para ampliar a acessibilidade. No entanto, a equiparação de oportunidades de acesso não garante necessariamente equiparação de oportunidades para aprender. Assim, como acontece na educação presencial, na EaD, o currículo deve ser planejado para atender à diversidade de aprendizes, respeitando suas limitações sensoriais e/ou cognitivas.

\section{Fase II - Design Universal para Aprendizagem}

Nessa fase, planejamos os cursos a distância que serão oferecidos no AVA. Um desses cursos será utilizado nas fases de testes, e o outro será aplicado de maneira formal aos participantes finais, que contribuirão efetivamente com os resultados da pesquisa (Ciclos III e IV). Estamos nesse momento desenvolvendo essa fase, na qual estamos nos concentrando na elaboração do material didático de um curso de Educação Financeira. 
Segundo Fernandes et al. (2013, p.25)

O Center for Applied Special Technology (Cast, 2011), uma organização de pesquisa e desenvolvimento, publicou um conjunto de princípios bastante flexíveis, que podem ser personalizados e ajustados às necessidades individuais, denominado Desenho Universal para Aprendizagem, destinado a orientar o desenvolvimento de um currículo que possa proporcionar a todos os indivíduos, especialmente aqueles com deficiências, igualdade de oportunidades para aprender. A nosso ver, essa é uma oportunidade para promover um movimento contrário ao usual, ou seja, ao invés de os alunos se adaptarem ao currículo, ele é que é adaptado às necessidades e/ou preferência dos alunos.

São nove os princípios do Desenho Universal para a Aprendizagem, divididos e apresentados em três eixos (Cast, 2011 apud Fernandes et al, 2013, pp. 25-26):

I. Utilização de múltiplos meios de representação do conteúdo.

1. Oferecer alternativas para a apresentação das informações.

2. Apresentar opções para o uso da linguagem, expressões matemáticas e símbolos.

3. Oferecer opções para a compreensão, orientando a visualização e a manipulação da informação.

II. Utilização de diversos meios de ação e expressão.

4. Proporcionar opções para a atividade física.

5. Oferecer alternativas para a expressão e a comunicação.

6. Orientar o estabelecimento de metas, de estratégias, de gerência da informação e dos recursos.

III. Oferecimento de diversas formas de engajamento.

7. Proporcionar opções para incentivar o interesse.

8. Oferecer suporte para sustentar as metas e os objetivos e promover a colaboração e o sentido de comunidade.

9. Apresentar opções para a autorregulação.

Apontamos que os princípios destacados nos três eixos podem ser utilizados pelo professor para planejar e elaborar suas aulas presenciais (Fernandes et al., 2013). 
As pessoas que possuem limitações sensoriais, ao utilizarem o computador como uma ferramenta que favorece o seu aprendizado, têm ampliadas as oportunidades na educação inclusiva

Em relação a esses aspectos, entendemos que os eixos I e II apresentam alguns princípios adequados às nossas ideias. Assim, no momento de se planejar um ambiente virtual que seja acessível e que possa atender às demandas das pessoas com limitações sensoriais, devemos considerar três importantes aspectos: a acessibilidade, a usabilidade e a adaptabilidade (Fernandes et al., 2013).

Quando nos referimos à usabilidade, procuramos sempre trabalhar para tornar as interfaces mais "amigáveis, intuitivas e fáceis de serem utilizadas por qualquer pessoa" (Fernandes et al., 2013, p. 27). Ainda segundo os autores, a adaptabilidade se caracteríza por propiciar ao usuário a adaptação do sistema segundo sua percepção, auxiliando de forma personalizada em suas tarefas (Fernandes et al., 2013). Por fim, a acessibilidade, segundo Santos (2012, p.21), resumidamente, "significa disponibilizar diversos conteúdos de forma flexível a grupos de usuários heterogêneos"

Compreendemos a partir dessas breves descrições que quanto maior a acessibilidade e a adaptabilidade, maior a usabilidade e quanto maior a usabilidade e a adaptabilidade, maior a acessibilidade (Fernandes et al., 2013).

\section{- Ciclo III - Testes do AVA}

Fase I - Estudo Piloto

Nessa fase, conduziremos um curso de caráter experimental para testarmos a acessibilidade do AVA.

A análise desse estudo nos permitirá avaliar e remodelar o AVA para atender às necessidades do público que queremos atingir.

Fase II - Redesign do AVA

Faremos as adaptações necessárias e voltaremos a testar o AVA.

A análise desse estudo nos permitirá avaliar e remodelar o AVA para atender às necessidades do público que queremos atingir. Esse processo será realizado até que o AVA seja considerado adequado pelos usuários. 
- Ciclo IV - Realização do curso

Fase I - Realização do curso

Nessa fase, convidaremos pessoas que apresentem algum tipo de limitação sensorial, principalmente, cegos e surdos. Esse convite ocorrerá através de contatos que professores e alunos do programa de Pós-Graduação em Educação Matemática da Universidade Anhanguera possuem bem como fazendo o uso de redes sociais e visitas a escolas e instituições específicas. Deixamos claro que, embora nosso público-alvo seja composto de pessoas cegas e surdas, o curso estará aberto à participação de todos, respeitando-se o limite de vagas a ser estabelecido, bem como os pré-requisitos mínimos, que será igual para todos os candidatos.

Fase II - Análise dos dados

Os dados coletados nessa fase serão analisados, o que nos oferecerá parâmetros para a avaliação final do projeto, permitindo-nos verificar se nossos objetivos foram alcançados, se conseguimos apresentar um modelo de Educação a Distância que seja possível atender a todos.

\section{Perspectivas}

Hoje se fazem presentes discussões a respeito dos direitos $s$ das pessoas com necessidades especiais. No entanto, percebe-se que a pessoa portadora de deficiência tem encontrado grandes obstáculos à sua aceitação e participação na sociedade. Obstáculos esses promovidos por barreiras arquitetônicas, falta de formação e informação de professores e, acima de tudo, referentes à sua inserção no mercado de trabalho. Esses e outros pontos que ainda poderiam ser destacados têm delegado a essas pessoas papéis e posições muito aquém de suas potencialidades.

Podemos destacar que a EaD vem auxiliando na aprendizagem e promovendo a inclusão social das pessoas em todos os níveis da educação assim como é previsto no sistema educacional brasileiro. Nessa direção, estamos propondo um ambiente interativo no qual alunos regulares ${ }^{2}$, alunos cegos e alunos surdos possam discutir de 
forma colaborativa e cooperativa utilizando os recursos de interação que o ambiente propicia. Para isso, o design do AVA conta com recursos destinados a atender às particularidades de cada um dos usuários. Para os cegos, os leitores de tela e para os surdos, a presença de janelas em Libras. É importante ressaltar que não estamos excluindo o cego e o surdo do ensino regular, das aulas presenciais, mas, sim, propondo uma alternativa aos seus estudos e formação profissional.

\section{Agradecimentos}

Agradecemos à Coordenação de Aperfeiçoamento de Pessoal de Nível Superior (CAPES) que, através do Programa de Suporte à Pós-Graduação de Instituições de Ensino Particulares/PROSUP, concede uma bolsa de estudos, formalizada através do projeto 2190/2010, contribuindo de forma significativa para a pesquisa.

\section{Nota/Notas}

${ }^{1}$ Esta pesquisa está inserida em um projeto maior que está se desenvolvendo nos muros da Universidade Anhanguera de São Paulo no programa de Educação Matemática. Tal projeto tem como premissa apresentar um ambiente virtual de aprendizagem acessível. Ressalta-se que este projeto já proporcionou 0 desenvolvimento de duas dissertações de mestrado já concluídas, uma em fase de conclusão e um doutorado em andamento. Porém, existe a perspectiva de um aumento de pesquisas que englobam o uso de ambientes virtuais de aprendizagem e pessoas com limitações sensoriais.

${ }^{2}$ A expressão alunos regulares é usada neste texto para designar aqueles que não têm limitações sensoriais.

\section{Referências bibliográficas}

Borba, M.C.; Malheiros, A.P.S.; Zulatto, R.B.A. (2007). Educação a Distancia online. Belo Horizonte: Autêntica. 
Cast (2011). Universal Design for learning guidelines version 2.0. Wakefield, MA. Acesso em 10/11/2014, Disponível em http://www.udlcenter.org/aboutudl/udlguidelines

Cud - Universal Design (2012). North Caroline State University - Center for Universal Design, 2008. Disponível em: http://www.ncsu.edu/project/designprojects/udi/center-for-universal-design/the-principles-of-universal-design

Fernandes, S. H. A. A., Santos, C. E. R., Bezerra, C., EaD e Educação Matemática Inclusiva: desafios e possibilidades In: $O$ uso de chat e de fórum de discussão em uma educação matemática inclusiva.1 ed. Rio de Janeiro: EDUR, 2013, v.5, p. 19-36.

Greenwood, D. J. \& Levin, M., Reconstructing the Relationships between Universities and Society through Action Research. In: DENZIN. N.K. \& LINCOLN, Y. S. (Eds), Handbook of Qualitative Research. 2nd ed. Thousand Oaks, California: Sage Publications Inc., 2000, p.85-106.

Inep. Censo Escolar da Educação Básica de 2013. Acesso em 19/11/2014, disponível em

http://download.inep.gov.br/educacao basica/censo escolar/apresentacao/2014 Lapresentacao coletiva censo edu basica 022014.pdf.

Lévy, P. As tecnologias da inteligência: o futuro do pensamento na era da informática. Trad. de Carlos Irineu da Costa. Coleção Trans. Rio de Janeiro: Ed. 34, 1993.

Martins, V. Quem necessita de Educação Especial? 2002. Disponível em: http://www.cvdee.org.br/evangelize/pdf/1 0383.pdf.

Santos, C. E. R. Interações de Aprendizes Cegos em Fórum de Discussão de um Ambiente Virtual de Aprendizagem Matemática. Dissertação de Mestrado, Universidade Bandeirante de São Paulo (UNIBAN), SP, Brasil, 2012. 